Classification

Physics Abstracts

$61.16 \mathrm{~B}$

\title{
Resolution of Semiconductor Multilayers using Backscattered Electrons in Scanning Electron Microscopy
}

\author{
Donato Govoni $\left({ }^{1}\right)$, Pier Giorgio Merli $\left({ }^{1}\right)$, Andrea Migliori $\left({ }^{1}\right)$ and Michele Nacucchi $\left({ }^{2}\right)$ \\ ( $\left.{ }^{1}\right)$ CNR-Istituto LAMEL, Via Piero Gobetti n.101, 40129 Bologna, Italy \\ $\left(^{2}\right)$ PASTIS-CNRSM SCpA, S.S.7 km 714.3, 72100 Brindisi, Italy \\ (Received March 5; accepted April 10, 1995)
}

\begin{abstract}
Observations of semiconductor multilayers with backscattered electrons in a scanning electron microscope have been used to revisit the concept of resolution of the backscattering imaging mode. With the support of Monte Carlo simulations of beam specimen interaction, it has been possible to achieve the following conclusions. All the backscattered electrons positively contribute to the image formation independently of their trajectories and specimen exit points. The generation volume does not represent in itself a limit to the resolution, which depends only on the beam size and the signal to noise ratio.
\end{abstract}

\section{Introduction}

The spatial resolution of the Scanning Electron Microscope (SEM) is considered to be limited by the following main factors: i) the electron probe diameter $(\Phi)$; ii) the size of the generation volume $\left(V_{\mathrm{g}}\right)$ for the detected signal; iii) the signal to noise ratio $(\mathrm{S} / \mathrm{N})$.

On the basis of the previous statement, the resolution of Backscattered Electron (BSE) images can be considered equal to the Full With Half Maximum (FWHM) of the BSE emission profile. This profile is the convolution of the intensity distribution in the electron probe with the spatial exit distribution of the BSE. As a consequence the spatial resolution of the image is limited by the electron range and high resolution should be possible in two ways: i) operating at very low energy; ii) using the low loss electron method, i.e. by collecting the electrons leaving the specimen after a single high angle $\left(>90^{\circ}\right)$ elastic scattering event (labelled BSE1) and suppressing the noise associated with diffused scattered ones (labelled BSE2) [1].

Since 1988 some experimental data [2-6], concerning the observation of multilayers with BSE, using an in lens field emission SEM, demonstrated a resolution of 2-3 $\mathrm{nm}$ (of the order of the beam spot size), at energies of $15-30 \mathrm{keV}$, although the electron range was several microns. These results cannot be explained within the previous model and indicate that the fundamental assumptions on the resolution in BSE imaging mode should be revisited.

Now we will analyse the causes of the difficulties in the interpretation of the images within the previous scheme and we will outline the approach that has been followed in order to overcome them. 
The scheme usually employed to define resolution refers to an homogeneous specimen that, by itself, does not provide any contrast, so that its use is somewhat misleading. It is necessary to make reference to an inhomogeneous specimen; i.e. a specimen actually producing a contrast in order to clarify the role of $\Phi, V_{\mathrm{g}}$ and $\mathrm{S} / \mathrm{N}$ in the image formation. The observation of multilayered structures based on III-V semiconductors, which can be easily produced by molecular beam epitaxy with a careful control of layers thickness and composition, represents the easiest approach to the study of resolution with an inhomogeneous specimen.

In an inhomogeneous specimen the irradiation of a detail $\mathrm{A}$ in a region $\mathrm{B}$ does not affect the production of BSE1 only; also the number of BSE2 depends on the presence and the features of the detail A. The detector does not "see" any emission profile; it just counts the total number of electrons and ignore their origins. Therefore, the possibility to resolve a specimen detail A in a region $B$ depends, besides on the beam spot size, on the difference in the total number of BSE (BSE1+BSE2) counted by the detector when the beam is positioned in A or B independently of their trajectories, i.e. independently of the signal generation volume and electron emission profile.

\section{Theoretical Results}

The last assumption can be quantified in the following way. Let us consider a bulk homogeneous specimen $B$ with an electron beam normally impinging on its surface. Now, let us imagine to replace with a material $\mathrm{A}$, having a different mean atomic number, a part of the specimen $\mathrm{B}$ centered around the beam impact point.

Let $\eta_{\mathrm{B}}$ and $\eta_{\mathrm{B}}^{\prime}(r)$ be the backscattering coefficient and the spatial exit density distribution function of BSE of the homogeneous sample. Let $\eta_{\mathrm{A}}$ and $\eta_{\mathrm{A}}^{\prime}(r)$ be the corresponding quantities of the inhomogeneous specimen (a detail $\mathrm{A}$ in a region $\mathrm{B}$ ). The integral

$$
\int_{x}^{+\infty}\left(\eta_{\mathrm{B}}^{\prime}(r)-\eta_{\mathrm{A}}^{\prime}(r)\right) \mathrm{d} r
$$

where $r$ and $x$ are radial distances, provides the difference in the number of BSE emerging beyond a distance $x$ from the center of the beam spot. Normalizing to $\eta_{\mathrm{B}}-\eta_{\mathrm{A}}$ we define the following function

$$
g(x)=\frac{\int_{x}^{+\infty}\left(\eta_{\mathrm{B}}^{\prime}(r)-\eta_{\mathrm{A}}^{\prime}(r)\right) \mathrm{d} r}{\eta_{\mathrm{B}}-\eta_{\mathrm{A}}} .
$$

The function $g(x)$ has a maximum equal to one at the origin, i.e. at the beam impact point, then it decreases to zero. The $x$ value where $g(x)$ vanishes defines a sort of "correlation distance" $r_{0}$. In the regions $x<r_{0}$ the exit probability of BSE is changed as a consequence of the presence of the detail $\mathrm{A}$, i.e. there is a correlation between a variation of the density material at the origin and the exit probability at a distance $x$ from it; for $x>r_{0}$ the exit probability is unchanged.

Monte Carlo (MC) simulations [7] have been performed for two different geometries: a GaAs homogeneous bulk sample and a GaAs/AlAs $(40 \mathrm{~nm}) / \mathrm{GaAs}$ bulk one using an electron probe having a diameter of $12 \mathrm{~nm}$ accelerated at $30 \mathrm{keV}$. The total number of electron trajectories was $1.1 \times 10^{5}$. The histogram bar widths of both BSE spatial density distributions are large enough to give a relative error of BSE number less than $10 \%$, whereas the relative error which affects $\eta_{\mathrm{B}}-\eta_{\mathrm{A}}$ is about $14 \%$. On the basis of these accuracy considerations, from the Figure 1 ( $g$ plot) a $r_{0}$ value approximately equal to $1.60 \mu \mathrm{m}$ can be deduced. For $x>r_{0}$ the variations of BSE number due to 


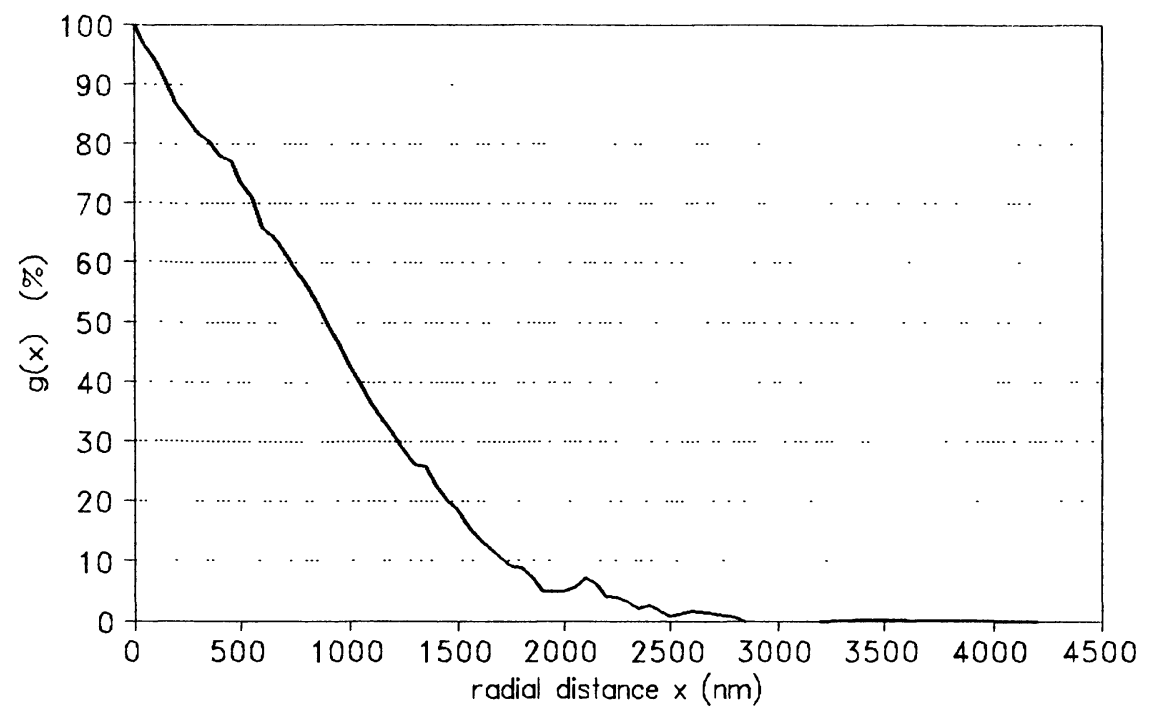

Fig. 1. - Behaviour of $g(x)$ vs. $x$. The maximum value of $g$ is normalized to $100 \%$.

the noise mask the effects of the presence of the detail A. It is worth noting that this value is $\approx 40$ times greater than the layer width and comparable to the electron range.

This result means that the presence of the detail A affects the number of BSE2 exiting from radial regions located up to a distance comparable to the electron range; then all the BSE provide information about the presence of the detail itself. Therefore, the complete collection of all BSE (BSE1+BSE2) improves the $\mathrm{S} / \mathrm{N}$ ratio and as consequence, increases the visibility.

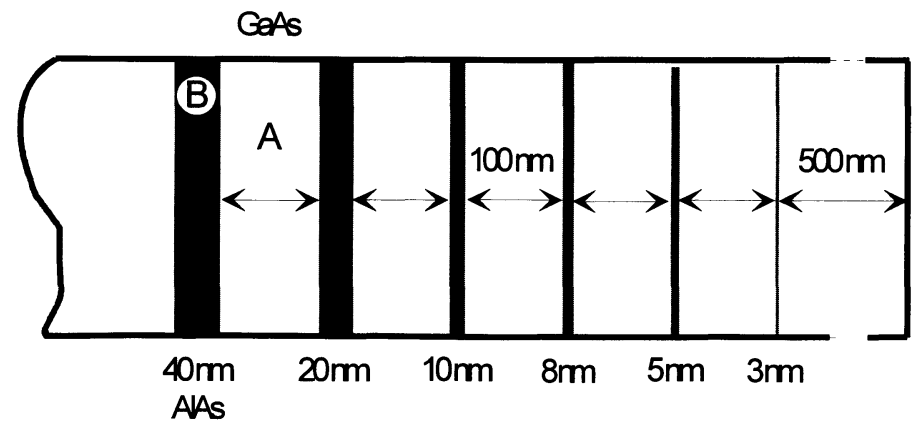

Fig. 2. - Scheme of the specimen geometry. A, B, indicate the beam impact points used in the simulation to deduce $\eta$. The same arrangement is adopted for each AlAs layer.

\section{Experimental Results and Discussion}

The specimen, observed in cross section, consists of AlAs layers having a thickness of 40, 20,10, 8, 5, $3 \mathrm{~nm}$ separated by $100 \mathrm{~nm}$ thick GaAs layers (Fig. 2). BSE images has been made using a 
Philips XL30 SEM equipped with $\mathrm{LaB}_{6}$ source. A standard BSE detector has been used. The beam current was in the range 4-80 pA. The beam current was deduced from specimen current, taking into account that the mean backscattering coefficient $\eta$ has a value of about $30 \%$. The picture point time of all the images was $1.3 \mathrm{~ms}$.

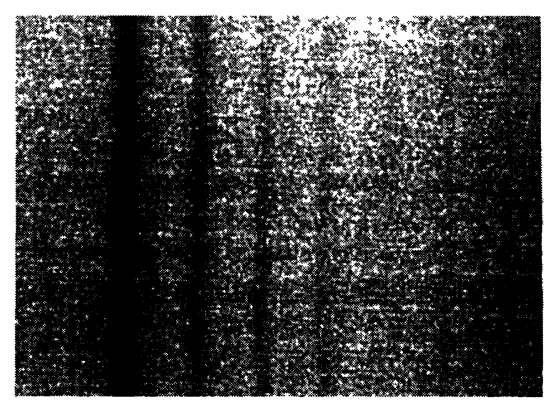

a

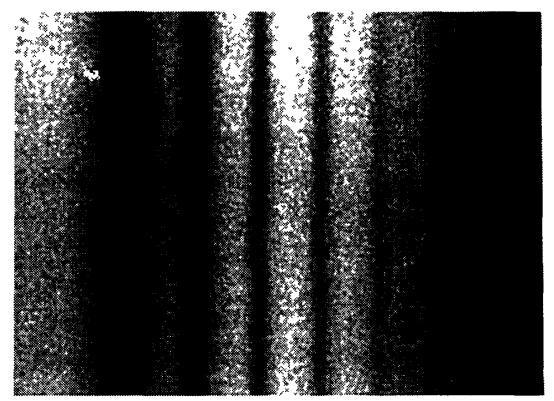

b

\section{$400 \mathrm{~nm}$}

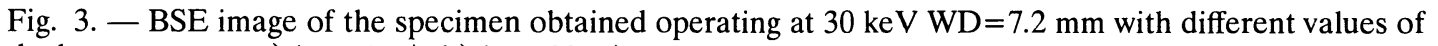
the beam current: a) $\left.i_{\mathrm{b}}=4 \mathrm{pA} ; \mathrm{b}\right) i_{\mathrm{b}}=80 \mathrm{pA}$.

In Figure 3 are reported two BSE images of the sample obtained at $30 \mathrm{keV}$ with a constant working distance (WD) of $7.2 \mathrm{~mm}$ at two different values of the beam current $i_{\mathrm{b}}=4 \mathrm{pA}$ (Fig. $3 \mathrm{a}$ ) and $80 \mathrm{pA}$ (Fig. $3 \mathrm{~b})$. In spite of the large value of the range $(R \cong 5 \mu \mathrm{m})$ the thin AlAs layers appear as dark lines separated by white ones which refer to the $\mathrm{GaAs}$ regions. Four lines are visible at $i_{\mathrm{b}}=4 \mathrm{pA}$, while all the six AlAs layers can be seen in the image taken at $i_{\mathrm{b}}=80 \mathrm{pA}$, showing that the number of visible lines increases with the beam current, i.e. with the improvement of $\mathrm{S} / \mathrm{N}$ ratio. According to Philips data the beam diameter is $\Phi \cong 3 \mathrm{~nm}$ for $i_{\mathrm{b}}=4 \mathrm{pA}$ and $\Phi \cong 12 \mathrm{~nm}$ for $i_{\mathrm{b}}=80 \mathrm{pA}$. Then the image of Figure $3 \mathrm{~b}$ shows that it is possible to detect specimen details having a spatial extension smaller than the beam diameter. Obviously, the dimension of the smaller lines are not related to the effective layer width (the lines are detected but not resolved) but are equal to the diameter of the electron probe; moreover the contrast decreases with the thickness of the layer. 
The experimental results have been compared with theoretical ones concerning the MC simulation of the electron interaction with the GaAs/AlAs multilayer. The Monte Carlo code [7] has been used to calculate the backscattering coefficient $\eta$ for an electron beam having a normal incidence to a cross section of the specimen. The beam energy was $30 \mathrm{keV}$ and the beam impact point was located at the centre of the GaAs (A) and AlAs (B) layers (see Fig. 2). For each impact point $N=7 \times 10^{4}$ events have been simulated. The electron probe has been assumed having a FWHM $\Phi$ equal to $3 \mathrm{~nm}$ and $12 \mathrm{~nm}$. The collection efficiency has been assumed equal to 1 , i.e all the backscattered electrons are considered to be collected by the annular detector. This hypothesis produces theoretical results that just slightly overestimate the number of BSE collected in the experiments because of the geometry of the detection [8].

Table I. - Contrast and minimum beam current for different layer thickness and for two beam diameters: a) $\Phi=3 \mathrm{~nm}, b) \Phi=12 \mathrm{~nm}$. It is appropriate to remark that the value of $\mathrm{C}$ and $i_{\mathrm{b}}$ minimum are affected by a large error (not reported). Especially for the smallest AlAs layer thickness.

\begin{tabular}{|c|c|c|c|c|}
\hline$t(\mathrm{~nm})$ & $\mathrm{a}) C \%$ & a) $i \min (\mathrm{pA})$ & $\mathrm{b}) C \%$ & $\mathrm{~b}) i \min (\mathrm{pA})$ \\
\hline 40 & 9 & 0.7 & 7 & 1.1 \\
20 & 7 & 1.1 & 6 & 1.4 \\
10 & 6 & 1.4 & 4 & 3.2 \\
8 & 5 & 2.1 & 3 & 5.8 \\
5 & 4 & 3.8 & 2 & 13 \\
3 & 3 & 6.2 & 1 & 52 \\
\hline
\end{tabular}

The values of the contrast $C$ for $\Phi=3 \mathrm{~nm}$ and $\Phi=12 \mathrm{~nm}$ with the corresponding values of the threshold beam current are reported in Table I. The contrast has been calculated according to the relation $C=1-\eta_{\mathrm{A}} / \eta_{\mathrm{B}}, \eta_{\mathrm{A}}<\eta_{\mathrm{B}}$; while the threshold current has been estimated according to the relation $i_{\mathrm{b}}>\frac{2 e t_{\beta}^{2}}{\tau \eta C^{2}}$ [7] where $e$ is the magnitude of electron charge, $\tau$ is the picture point time and $t_{\beta}$ is a numerical factor that, for a confidence level of $99 \%$ is equal to 2.57 .

The values of the theoretical threshold current are comparable with the experimental ones leading to a consistent interpretation: for $i=4 \mathrm{pA}(\Phi \cong 3 \mathrm{~nm})$ only four AlAs layers can be observed (Tab. Ia), whereas for $i=80 \mathrm{pA}(\Phi \cong 12 \mathrm{~nm})$ all the AlAs layers can be detected (Tab. 1b).

It is necessary to remark that in the calculations only the number of BSE is taken into account, while the exit points of the BSE trajectories are completely ignored. It means that, in order to explain the experimental results, the relevant point is not the spatial exit distribution of the BSE, but just the different number of BSE produced when the electron beam impinges on AlAs or GaAs layers.

\section{Conclusions}

The observations of the semiconductor multilayers reported in this paper allow to revisit the concept of resolution achievable with BSE. In fact, it is shown that the generation volume does not 
represent in itself a limit to the resolution, which depends only on the beam size and the S/N ratio. The collection of the BSE2 does not deteriorate the resolution but improves the $\mathrm{S} / \mathrm{N}$ ratio and, consequently, the visibility is increased.

\section{References}

[1] Wells O.C. and Nacucchi M., in Proc. Int. School on Electron Microscopy in Materials Science, P.G. Merli and M. Vittori Antisari Eds. (World Scientific, Singapore, 1992) p. 479.

[2] Ogura K., Proc. IVth Asia Pacific Conference and Workshop on Electron Microscopy (1988) p. 165.

[3] Ogura K. and Kersker M.M., Proc. 46th Annual Meeting of the Electron Microscopy Society of America (San Francisco Press, San Francisco, 1988) p. 204.

[4] Franchi S., Merli P.G., Migliori A., Ogura K. and Ono A., Proc. of XIIth Int. Congr. Electr. Microsc., Vol. 1 (San Francisco Press, San Francisco, 1990) p. 380.

[5] Ogura K., Ono A., Franchi S., Merli P.G. and Migliori A., Proc. of XIIth Int. Congr. Electr. Microsc., Vol. 1 (San Francisco Press, San Francisco, 1990) p. 404.

[6] Ogura K., JEOL News 29 (1991) 6.

[7] Merli P.G. and Nacucchi M., Ultramicrosc. 50 (1993) 83.

[8] Bosacchi A., Franchi S., Govoni D., Mattei G., Merli P.G., Migliori A. and Nacucchi M., Mater. Res. Soc., Proc. of the Symp. A 354 (1994) to be published. 